\title{
Measurement Of Thermal Contact Resistance Between The Mold And The Polymer For The Stretch-blow Molding Process
}

\author{
M. Bordival ${ }^{1}$, F.M. Schmidt ${ }^{1}$, Y. Le Maoult ${ }^{1}$, E. Coment ${ }^{2}$ \\ ${ }^{I}$ CROMeP - Ecole des Mines d'Albi Carmaux - Campus Jarlard - 81000 Albi, France \\ ${ }^{2}$ NEOTIM Company - Albisia, 54 rue Gustave Eiffel - 81000 Albi, France
}

\begin{abstract}
In the stretch-blow molding process, the heat transfer between the polymer and the mold is of prime interest. Although the time of contact is very short (typically around $0.5 \mathrm{~s}$ ), the heat transfer affects the mechanical properties of the bottle, and the quality of final parts. In order to model heat transfers at the interface, a classical approach - generally adopted in numerical softwares - is to impose the heat flux density boundary condition thanks to a parameter called Thermal Contact Resistance (TCR). This paper focuses on describing the experimental method developed in order to measure evolution of this thermal parameter (TCR) versus time, as well as results obtained on the CROMeP blowing machine. In this study, a mold has been instrumented with two different sensors. The first probe allows to estimate the heat flux density and temperature at the mold surface temperature, using a linear inverse heat condution problem (Function Specification Method). The second device is used to measure the surface temperature of the PET during the blowing. This measurement is non intrusive, and can be applied within an industrial environment during the blowing step. In addition, air pressure inside the preform is also measured during the blowing. This work is part of the European project "APT_PACK" (Advanced knowledge of Polymer deformation for Tomorrow's PACKaging).
\end{abstract}

Keywords: stretch-blow molding process, thermal contact resistance, inverse problem.

PACS: 44.10. $+\mathrm{i} ;$ 44.40. $+\mathrm{a} ; \mathbf{4 4 . 2 0 . + b}$

\section{INTRODUCTION}

During a stretch-blow molding cycle, the PET preform is successively blown and maintained into contact with an aluminum mold, thanks to air pressure. The polymer is cooled down by the die, and it rigidifies inside the cavity. In such process, the solidification time of the part is controlled by the heat transfer between the plastic and the mold. The PET cooling rate affects not only the process efficiency, but also mechanical properties of the final bottle. For these reasons, the PET cooling rate prediction is of prime interest, and can not be carried out without a precise understanding of the heat transfer properties. Owing to the imperfect nature of contact between plastic part and mold inner surfaces, the heat transfer is affected by a Thermal Contact Resistance (TCR). The TCR is generally used in numerical software in order to impose the heat flux density boundary condition. Consequently its value is critical in the aim of achieving accurate simulations. However this parameter is often badly estimated. A few researchers have reported values of TCR at plastic-metal interfaces 
from steady state experiments [1]. However, the TCR in Stretch-Blow Molding Process (SBMP) is an unsteady phenomenon, and one cannot simply extend steady state results to such a transient process. Evolution of TCR with time was abundantly studied for different processes like casting die [2, 3], or injection-molding [4-6]. Some researchers have proposed analytical model allowing to link TCR to the pressure [6]. However in such processes, the nature of contact is quiet different since the material is molten. Moreover applied pressures and temperature ranges are strongly higher than for the SBMP. In the present work, we have investigated the efficiency of the method exposed in $[2,4]$ for the SBMP. For that, specific sensors have been developed in order to measure at the same time, the evolution of the TCR and the air pressure inside the preform. Main difficulties related to this measurement are the very short time of the phenomenon (around $0.5 \mathrm{~s}$ ) as well as the small temperature increases which must be detected (typically some degrees for the mold).

\section{MEASUREMENT STRATEGY AND PROCEDURE}

An imperfect contact between two materials gives rise to the so-called Thermal Contact Resistance, characterized by a sharp drop in temperature at the interface. It may be defined per surface unit as:

$$
T C R=\frac{T_{P E T}-T_{\text {Mold }}}{\varphi}
$$

where $T_{P E T}$ and $T_{\text {Mold }}$ are respectively polymer and mold surface temperatures, and $\varphi$ is the heat flux density exchanged. The TCR (in $\mathrm{m}^{2} . \mathrm{K} \cdot \mathrm{W}^{-1}$ ) is also the inverse of the heat transfer coefficient $h$. In order to compute the TCR, the strategy adopted in this study is to determinate $\varphi, T_{P E T}$, and $T_{\text {Mold. }}$.

\section{Measurement Procedure for the Polymer Surface Temperature}

The strategy adopted for this measurement is to use an infrared pyrometer, inserted through the mold thickness, in middle-height. This type of measurement has the advantage of being non-intrusive. However, it can't be suitably performed without a precise knowledge of PET optical properties. In order to ensure that the pyrometer measures a surface temperature, the PET must behave like an opaque body. For that, the pyrometer spectral range must be chosen judiciously. Moreover the PET mean emissivity over the considered spectral range is required for the sensor calibration.

In this study, optical properties of PET Tergal F9 have been measured with a Perkin Elmer FT-IR spectrometer, over the range $2.5-25 \mu \mathrm{m}$. The method consists in determining both spectral specular reflection coefficient $\rho_{\lambda}$, and transmission coefficient $\tau_{\lambda}$. Reflection measurements are performed on thick samples $(2 \mathrm{~mm}$ for instance), while transmission ones are realized for different thicknesses. At the thermal equilibrium, the spectral emissivity $\varepsilon_{\lambda}$ is deduced form the Kirchhoff law:

$$
\varepsilon_{\lambda}=1-\rho_{\lambda}-\tau_{\lambda}
$$


For an opaque body $\left(\tau_{\lambda}=0\right)$, the spectral emissivity can be easily determinated by measuring the reflection coefficient. Finally, the mean emissivity $\varepsilon$ defined as following can be computed:

$$
\varepsilon\left(T_{P E T}\right)=\frac{\int_{\Delta_{\lambda}} \varepsilon_{\lambda} I_{\lambda}^{\circ}\left(T_{P E T}\right) d \lambda}{\int_{\Delta_{\lambda}} I_{\lambda}^{\circ}\left(T_{P E T}\right) d \lambda}
$$

where $I_{\lambda}^{\circ}\left(T_{P E T}\right)$ is Planck's Intensity [7] at PET temperature. Full details about this procedure are reported in [8]. Results are illustrated FIGURE 1.
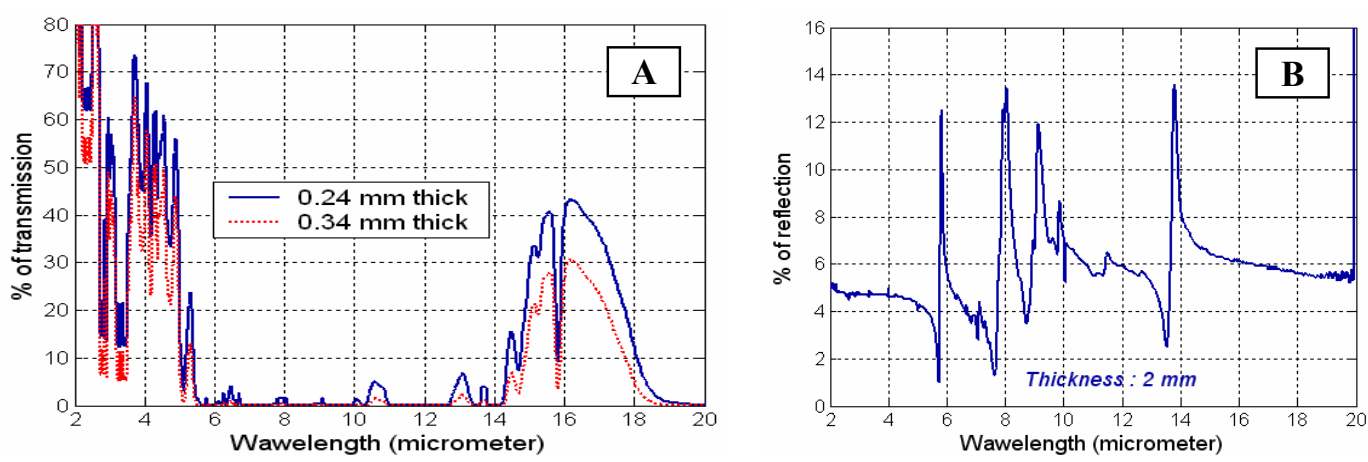

FIGURE 1. A: Spectral transmission coefficient of PET Tergal F9 for two sample thicknesses B: Spectral reflection coefficient of PET Tergal F9 for a $2 \mathrm{~mm}$ thick sample

FIGURE 1A reveals that PET presents successively transparent and opaque regions between $1 \mu \mathrm{m}$ and $20 \mu \mathrm{m}$. The range $8-14 \mu \mathrm{m}$ is particularly interesting since the transmission coefficient is less than $5 \%$, even for relatively thin samples $(0.24 \mathrm{~mm})$. For this reason we have chosen to compute the mean emissivity over this range. Results are illustrated FIGURE 2A. We observe that for a thickness higher than 0.3 $\mathrm{mm}$, the mean emissivity reaches an asymptotic value equal to 0.93 . This characterizes an opaque body for which the emission is a surface phenomenon.

As a conclusion these results demonstrate that a pyrometer operating in the range 8$14 \mu \mathrm{m}$ will measure the PET surface temperature, provided that the preform thickness is sufficiency large. For this study, a pyrometer Optris ${ }^{\circledR}$ CTfast $8-14 \mu \mathrm{m}$ has been chosen (FIGURE 2B). The sensor response time, given by the constructor, is equal to $17 \mathrm{~ms}$, which is sufficiently small regarding to the typical time of contact $(0.5 \mathrm{~s})$.
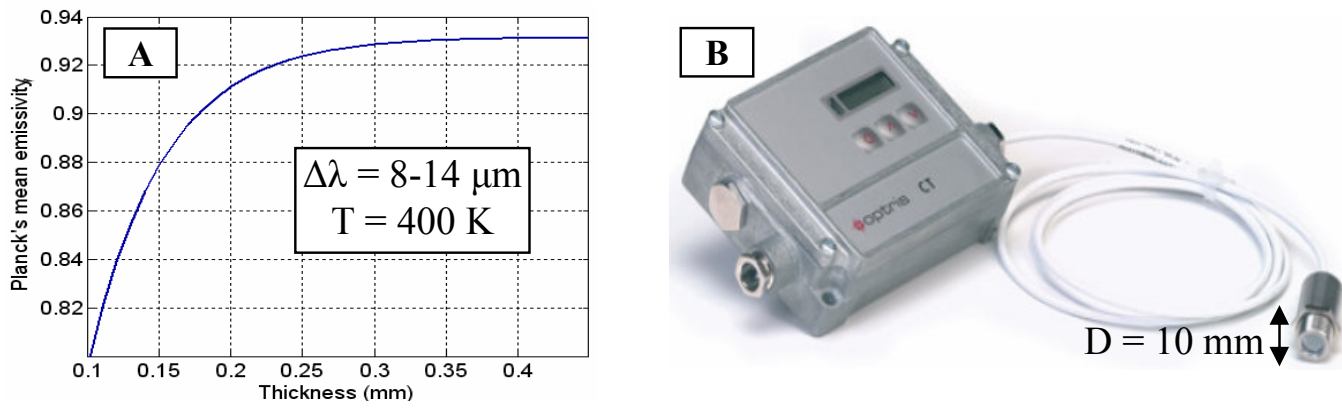

FIGURE 2. A: Planck mean emissivity of PET Tergal F9 versus sample thickness B: Pyrometer Optris ${ }^{\circledR}$ CTfast 8-14 $\mu \mathrm{m}$ 


\section{Measurement of Heat Flux Density and Mold Surface Temperature}

The second sensor developed for this application (FIGURE 3A), is inserted at the same height position as the pyrometer, but on the opposite side of the mold (FIGURE 3B). Its external dimensions are the same as a Kistler pressure sensor to make its installation in dies easy. It is realized in the same aluminum as the mold in order to avoid thermal disturbances. Therefore, the heat flux is considered as unidirectional through the mold thickness, assumption necessary for the inverse method. The measurement is realized thanks to two K-type micro-thermocouples, brazed into the gauge at different depth, and perpendicularly to the heat flux direction. Critical design features that are essential to an accurate estimation of the heat flux are discussed in [2]. The method is based on the linear inverse heat condution problem (Function Specification Method), fully described in [9].

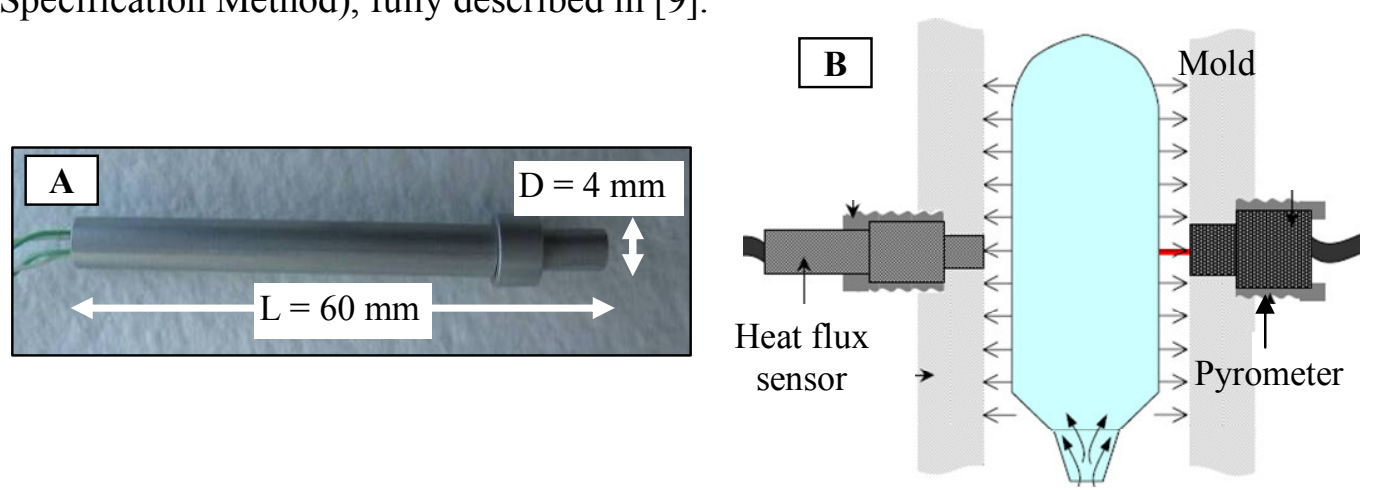

FIGURE 3. A: Heat flux density sensor - B: Location of sensors through the mold

\section{Experimental Device}

Trials were performed on the CROMeP blowing machine (FIGURE 4A), with APT_PACK $18.5 \mathrm{~g}$ preforms (FIGURE 4B), injected from PET Tergal F9. The preform heating is ensured by an infrared oven composed of six halogen lamps of nominal power $1 \mathrm{~kW}$. The air pressure inside the preform is measured using a Kulite LE 125 sensor (FIGURE 4B). Data are recorded thanks to a Nimtech acquisition system.
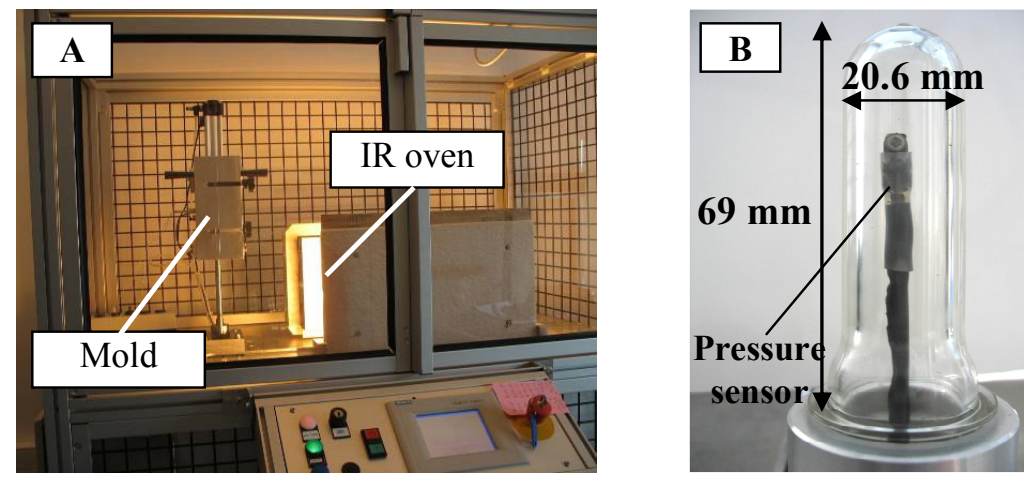

FIGURE 4. A: CROMeP blowing machine - B: APT_PACK $18.5 \mathrm{~g}$ preform and pressure sensor 


\section{RESULTS AND DISCUSSION}

During the blowing step (without any stretch rod), the air pressure inside the bottle follows typical variations. In the first time, the pressure increases sharply. As soon as the pressure is sufficient to blow the preform, the bottle volume increases and consequently the air pressure drops. While preform internal volume remains constant, the pressure reaches gradually its nominal value. This typical evolution of air pressure gives a good representation of the blowing kinematic, and remains a reference in order to understand variations of measured temperatures. FIGURE 5A illustrates the PET surface temperature. This one starts to increase before the beginning of the blowing. This is probably due to the own emission of the preform, which is already inside the mold. The pyrometer receives radiation from the preform, after different reflexions inside the cavity. At approximately $2 \mathrm{~s}$ we observe a second temperature rise of approximately $14{ }^{\circ} \mathrm{C}$. This one is more difficult to explain, and may be due to different phenomena: 1 - The temperature increases because of the polymer viscous dissipation, which appears during the stretching. 2- The temperature rise is due to an overestimating of the polymer emissivity, and in other words, to a measurement error. The thickness of the final bottle was measured in middle height. Its value is equal to 0.2 $\mathrm{mm}$. Regarding to FIGURE 1A, the emissivity decrease is relatively small, and should not be sufficient to justify the temperature increase. As a conclusion, we can assume that the temperature increase is probably due to a combination of both phenomena.
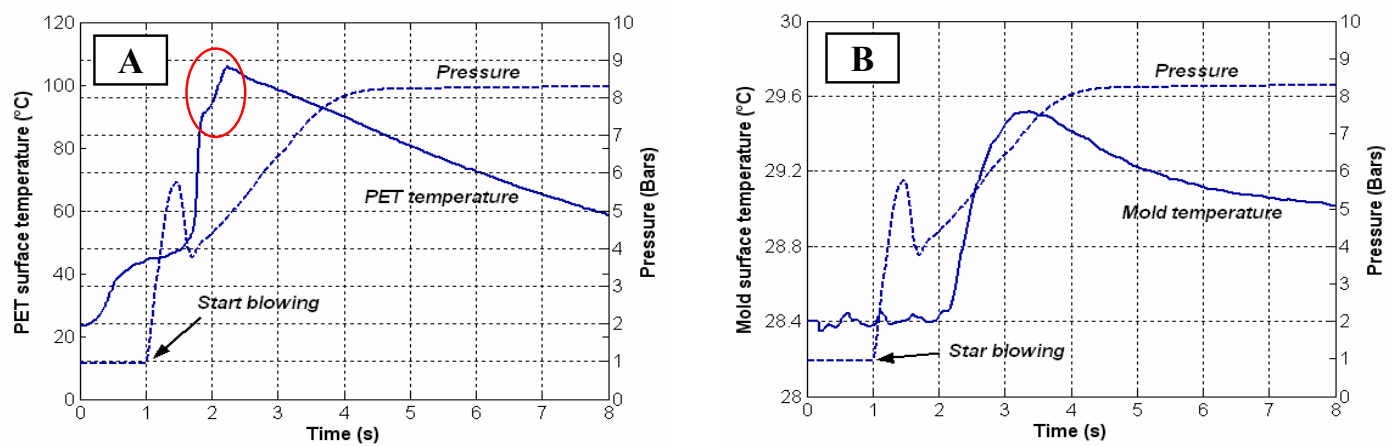

FIGURE 5. A: Measured PET surface temperature and air pressure versus time B: Computed mold inner surface temperature and pressure versus time

FIGURE 5B illustrates evolution of the mold surface temperature versus time, computed using the inverse method. We observe that for one blowing cycle, the temperature increase is around $1{ }^{\circ} \mathrm{C}$. For one-shot bottle, this allows to verify the isotherm assumption generally adopted in numerical software to model heat transfer inside the mold. FIGURE 6A illustrates the heat transfer coefficient (inverse of the TCR) versus time. This one drops dramatically in the first second following the peak value, while the air pressure still increases. This phenomenon was observed by different investigators for other processes [2, 4]. It is generally admitted that this sudden rise of TCR (FIGURE 6B) is related to the air gap due to polymer shrinkage. However evolution of contact should be quiet different for the SBMP, since PET does not change of phase. A more probable assumption could be that the polymer cooling is slowed down by the air gap between the pyrometer and the bottle. 

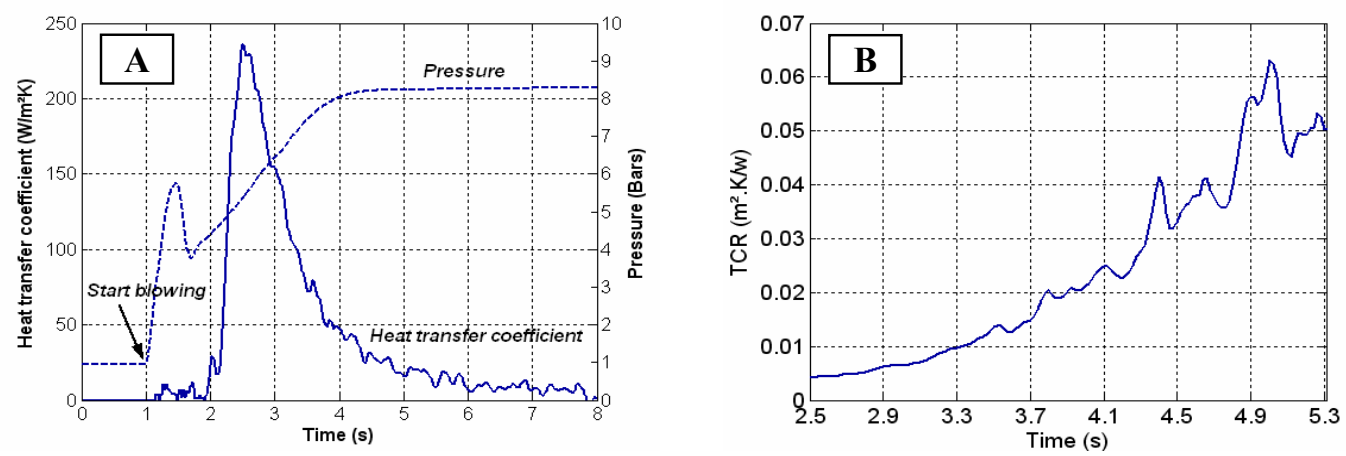

FIGURE 6. A: Heat transfer coefficient and air pressure versus time -

$\mathrm{B}$ : Corresponding TCR versus time over the range $2.5-5.3 \mathrm{~s}$

The peak value of the heat transfer coefficient (averaged on five trials) is equal to $225 \mathrm{~W} \cdot \mathrm{m}^{-2} \cdot \mathrm{K}^{-1}$, which corresponds to a TCR equal to $0.0044 \mathrm{~m}^{2} . \mathrm{K}^{-\mathrm{W}^{-1}}$. The standard deviation of this peak value is equal to $20 \mathrm{~W} \cdot \mathrm{m}^{-2} \cdot \mathrm{K}^{-1}$ (9\% error), which indicates that the repetitiveness is good.

\section{CONCLUSION AND PROSPECTS}

Preliminary results demonstrate that response time of sensors is sufficiently fast to measure the variation of the heat transfer coefficient in a very short time $(0.5 \mathrm{~s})$, corresponding to real process conditions. Typical values of TCR that were determinated in this study should be helpful for more accurate simulation of the stretch-blow molding process. However measurements have been performed with constant process conditions. The air pressure as well as the preform temperature should have an important impact on the TCR. Future work will focus on the influence of such parameters.

\section{ACKNOWLEDGMENTS}

This work war carried out as a community STRP project named APT_PACK, contact $\mathrm{n}^{\circ}$ STRP 505204-1. Authors would thank Logoplast Company for injecting preforms used in this work, as well as E. Coment and V. Lucin for their contribution.

\section{REFERENCES}

1. L. S. Fletcher, Experimental Heat Transfer, Fluid Mechanics and Thermodynamics, Kelleher, ed., Elsevier Science Publishers B.V, 1993.

2. G. Dour and M. Dargusch, Journal of Materials Processing Technology 169, 223-233 (2005).

3. A. Hamasaiid and al., MCWASP Conference Proceedings XI, TMS, Opio, 2006, pp. 1205-1212.

4. A. Bendada, and al., Applied Thermal Engineering 24, 2029-2040 (2004).

5. C. J. Yu, and al., Polymer Engineering and Science 30, 1599-1605 (1990).

6. H. Massé, "Les couplages thermomécaniques lors de la solidification de matériaux polymères", Ph.D. Thesis, Université de Bordeaux, 2000.

7. M.F. Modest, Radiative Heat Transfer, McGraw-Hill, Inc, 1993.

8. S. Monteix, Y. Le Maoult, F.M. Schmidt, QIRT Journal, 2001.

9. J. Beck, Int. J. Heat Mass Transfer 13, 703-715 (1970). 